\title{
Die grenzenlose Verbreitung von Klein- und Leichtwaffen: Argumente für eine restriktive deutsche Rüstungsexport- politik
}

Die unkontrollierte Verbreitung von Klein- und Leichtwaffen bleibt eine der globalen Herausforderungen mit beträchtlichen Folgen für die Friedenskonsolidierung und die Prävention von Gewaltkonflikten. Der Forumsbeitrag zeigt, dass sich die illegale Proliferation von Klein- und Leichtwaffen oftmals aus legalen, zwischenstaatlichen Rüstungsexporten speist. Deutschland sollte seine nationale Rüstungsexportpolitik deshalb grundsätzlich überdenken, gerade was die Genehmigung von Rüstungsexporten und Lizenzvergaben von Klein- und Leichtwaffen an sogenannte Drittstaaten, vor allem auch im Nahen Osten, anbelangt. Die gegenwärtige Rüstungsexportpolitik bleibt in dieser Hinsicht widersprüchlich und ambivalent. Einerseits hat Deutschland als einer der ersten Staaten den internationalen Waffenhandelsvertrag ratifiziert, der den Handel mit konventionellen Rüstungsgütern weltweit entlang rechtlich verbindlicher Kriterien regulieren will. Andererseits exportiert Deutschland weiterhin Kriegswaffen in Konfliktgebiete oder auch an Staaten mit negativer Menschenrechtsbilanz.

Schlagworte: Rüstungskontrolle, Abrüstung, Schusswaffen, Gewalt, Vereinte Nationen

\section{Einleitung}

Die deutsche Rüstungsexportpolitik sorgt für Schlagzeilen. In den Fokus sind die Klein- und Leichtwaffen geraten. ${ }^{1}$ Besorgniserregend ist dabei ein Trend, der seit

1 Nach wie vor gibt es keine einheitliche internationale Definition, welche Waffenkategorien Kleinund Leichtwaffen umfassen. Die Definition des Expertenpanels der Vereinten Nationen (UN) zur Kleinwaffenproblematik von 1997 schlug eine weite Definition vor, die beispielsweise auch Revolver, Sportwaffen und Munition einschließt. Diese umfassende Definition konnte sich in den Verhandlungen des UN-Kleinwaffenaktionsprogramms von 2001 nicht durchsetzen: Munition, Revolver/Pistolen und Sportwaffen sind hier ausgenommen (Greene/Marsh 2012: 2). Der deutsche Rüstungsexportbericht verwendet den Kleinwaffenbegriff der Organisation für Sicherheit und Zusammenarbeit in Europa sowie der EU. Als Kleinwaffen werden militärische Handfeuerwaffen (Maschinenpistolen/-gewehre, Sturmgewehre und leichte Maschinengewehre) begriffen; Leichtwaffen sind von einer Person oder von Mannschaften tragbare leichte Waffen, insbesondere tragbare Raketen- und Artilleriesysteme. Der Rüstungsexportbericht (BMWi 2014 a: 27) verweist darauf, dass zivile Waffen wie Jagd- und Sportwaffen, aber auch Selbstverteidigungswaffen (Revolver/Pistolen) in diesen Definitionen und damit auch im Berichtswesen nicht eingeschlossen sind. 
2011 einen Anstieg von Kleinwaffenexporten an sogenannte Drittstaaten verzeichnet, also jene Staaten, die außerhalb der EU, der NATO und gleichgestellter Staaten wie der Schweiz oder Australien liegen. Auch die Zahlen des jüngsten Rüstungsexportberichts, der erstmals bereits im Juni 2014 vorgelegt wurde, dessen Genehmigungen jedoch noch von der ehemaligen Bundesregierung beschieden worden sind, unterstreichen diesen Negativtrend. Lagen die Einzelausfuhrgenehmigungen von Kleinwaffen 2011 noch bei 17,92 Millionen Euro, waren es 2012 37,09 Millionen Euro und 2013 42,23 Millionen Euro, die allein an Drittstaaten gingen (BMWi 2014 a: 28). Insgesamt sind 2013 Einzelausfuhrgenehmigungen von 82,63 Millionen Euro erteilt worden, womit der Anteil der Kleinwaffenexportgenehmigungen an Drittstaaten bei gut 51\% liegt. Größter Abnehmer deutscher Kleinwaffen ist Saudi-Arabien, das allein 2013 18.201 Gewehre und rund 96.000 Komponenten für Gewehre und weitere 80.000 Teile für Maschinenpistolen erhalten hat, um damit unter anderem die 2008 genehmigte Lizenzproduktion für das G36-Sturmgewehr gewährleisten zu können. ${ }^{2}$ Der im Oktober veröffentlichte Zwischenbericht der Bundesregierung über die Rüstungsexporte weist für das erste Halbjahr 2014 nun einen deutlichen Rückgang der Genehmigungen für Kleinwaffen auf. Sie beliefen sich auf 21,3 Millionen Euro, was im Vergleich zum 1. Halbjahr 2013 eine Verringerung um rund 18 Millionen Euro ausmacht (BMWi 2014 b: 8). Daraus jedoch eine Trendwende abzuleiten, erscheint zum jetzigen Zeitpunkt verfrüht. Immerhin kündigte das Bundeswirtschaftsministerium an, neue »Kleinwaffengrundsätze« für deutsche Rüstungsexporte zu erarbeiten.

Die Proliferation von Klein- und Leichtwaffen ist ein globales Problem mit erheblichen Folgekosten. Nur allzu oft beginnt die illegale Verbreitung dieser Waffen und ihrer Munition als legaler Rüstungsexport von Staat zu Staat, um in Drittländern Streitkräfte oder Polizei auszustatten. Unzureichende Sicherungsmaßnahmen solcher Waffenbestände, fragile Staatlichkeit, defizitäre Sicherheitssektoren, Korruption, Retransfers ${ }^{3}$ der Waffen aufgrund politischen Kalküls oder strategischer Interessen - die Gründe sind vielfältig, die aus legalen Exporten und staatlichen Arsenalen illegale Kleinwaffen werden lassen. Die direkten und indirekten Folgen der Kleinwaffenproliferation sind beträchtlich. Jährlich sterben im Durchschnitt 526.000 Menschen an den Folgen von Schusswaffengewalt. Nur etwa 10\% davon sind Opfer kriegerischer Gewalt; die große Mehrheit der Menschen wird infolge von Gewaltverbrechen getötet (SAS 2013: 7-15). Die indirekten Folgekosten solcher Proliferation von Klein- und Leichtwaffen sind vielfältig und ungleich

2 Für hilfreiche Kommentare und Anregungen danke ich Jan Grebe, Tina Schmitt, dem anonymen Gutachter sowie der Redaktion der Zeitschrift für Friedens- und Konfliktforschung.

3 Von »Retransfer« wird gesprochen, wenn die Waffenexporte nicht im Zielland verbleiben, sondern von diesem weiterverbracht werden. 
schwerer zu beziffern. Ein hohes Aufkommen an illegalen Kleinwaffen trägt zur Destabilisierung von Staaten und Gesellschaften bei. Beispiele zeigen, dass Kleinwaffen auf vielfältigen Wegen nahezu ungehindert in Konfliktgebiete gelangen und dort zur Gewalteskalation führen (SAS 2014: 215-218).

Seit mehr als zehn Jahren müht sich die internationale Staatengemeinschaft, Regelungen zur Bekämpfung des unrechtmäßigen Kleinwaffenhandels zu verabschieden und umzusetzen. ${ }^{4}$ Gerade weil die Thematik so komplex und diffizil ist, haben die Regelungen bislang zu keiner tiefgreifenden Änderung der globalen Proliferation von Kleinwaffen geführt. Die Hoffnung der internationalen Staatengemeinschaft ruht nun auch auf dem internationalen Waffenhandelsvertrag (Arms Trade Treaty, ATT), der nach 53 Ratifikationen im nächsten Jahr in Kraft treten wird. ${ }^{5}$ Deutschland engagiert sich seit Jahren nicht nur in der Umsetzung des Kleinwaffenaktionsprogramms (PoA) ${ }^{6}$ der Vereinten Nationen (UN) von 2001, sondern war auch ein Fürsprecher im Verhandlungsprozess über einen »starken und robusten« Waffenhandelsvertrag. Besonders das Kriterium der Menschenrechte als Bewertungsgrundlage für Entscheidungen über Rüstungsexporte wurde von deutscher Seite stets betont. Jedoch zeigt sich in der deutschen Rüstungsexportpolitik eine Diskrepanz zwischen dem eigenen internationalen Engagement und dem nationalen Handeln. Auch widerspricht der oben erwähnte Trend, Klein- und Leichtwaffen in immer stärkerem Umfang an solche Drittstaaten zu exportieren, deren Menschenrechtspolitik problematisch ist oder die den Endverbleib dieser Waffen nicht gewährleisten, einer wirklich restriktiven deutschen Rüstungsexportpolitik. Die Ambivalenz dieser Politik gipfelte erst kürzlich in der Entscheidung der Bundesregierung, Klein- und Leichtwaffen aus Bundeswehrbeständen an die kurdischen Peschmerga nach Nordirak zu liefern, um diese im Kampf gegen die Terrormiliz Islamischer Staat (IS) zu unterstützen und auszurüsten (Brost et al. 2014: 6).

Zwar handelt es sich dabei um keinen regulären Rüstungsexport, doch weicht Deutschland damit erneut vom Prinzip ab, keine Waffen in Krisen- und Kriegsge-

4 Im Englischen wird von »illicit trafficking of small arms and light weapons« gesprochen. Bei der Verabschiedung des Kleinwaffenaktionsprogramms (PoA) 2001 konnte sich die internationale Staatengemeinschaft nicht darauf einigen, auch legale Kleinwaffenexporte von Staat zu Staat mit in den Geltungsbereich des PoA einzubeziehen - viele Staaten wollten allein die illegale, nicht-staatliche Verbreitung von Klein- und Leichtwaffen verregeln und sahen sämtliche staatliche Waffenexporte als legal an. Um jedoch die Grauzonen von beispielsweise verdeckten staatlichen Waffenlieferungen miteinbeziehen zu können, einigte man sich auf den Ausdruck illicit, der in der Sprachregelung des Auswärtigen Amtes mit »unrechtmäßig« übersetzt wird (siehe dazu BMWi 2014 a: 2-3).

5 Zum aktuellen Stand der zuletzt 53 Ratifizierungen siehe: www.controlarms.org (letzter Zugriff: 2.10.2014).

6 Die Abkürzung PoA steht für Programme of Action to Prevent, Combat and Eradicate the Illicit Trade in Small Arms and Light Weapons in All Its Aspects. 
biete zu liefern. Dieser Forumsbeitrag will insgesamt für die direkten und indirekten Folgen der Verbreitung von Klein- und Leichtwaffen sensibilisieren, um darüber die Notwendigkeit einer Umkehr in der deutschen Rüstungsexportpolitik zu begründen. Hierfür wird in Kapitel 2 zunächst verdeutlicht, warum es trotz geschätzter 875 Millionen weltweit schon vorhandener Kleinwaffen so wichtig ist, eine verantwortungsvolle und restriktive deutsche Rüstungsexportpolitik zu betreiben. Dieses Kapitel wird einen Überblick über die Proliferationsgefahren geben. Kapitel 3 bewertet dann die direkten und indirekten Folgekosten der illegalen Kleinwaffenverbreitung. Kapitel 4 konzentriert sich auf die bisherigen schwierigen Bemühungen, globale Regelungen zu verhandeln und auf diese Weise die Proliferation von Klein- und Leichtwaffen einzudämmen. Kapitel 5 entwickelt abschließend Forderungen zur Umsetzung einer restriktiven deutschen Rüstungsexportpolitik im Hinblick auf Klein- und Leichtwaffen sowie Munition und knüpft damit an den Beitrag von Jan Grebe (2013) zur deutschen Rüstungsexportpolitik an.

\section{Vielfältige Gründe für die weltweite Verbreitung von Kleinwaffen}

Die Forschung zu den Ursachen und Gründen der Verbreitung von Kleinwaffen hat sich erst in den letzten 15 Jahren entwickelt. Auffällig ist dabei, dass frühere Erkenntnisse, beispielsweise in Form von Opferstatistiken durch Kleinwaffengewalt, inzwischen revidiert werden mussten. Frühere Schätzungen des Small Arms Survey sprachen von jährlich 500.000 Opfern, wovon allein 300.000 in Kriegen und Konflikten durch Schusswaffengewalt zu Tode kommen würden. Neuere Statistiken der World Health Organization zeigen, dass die Zahl der Opfer durch Kleinwaffen in Kriegen und Konflikten weitaus geringer ist (siehe oben). Solche Korrekturen sind insgesamt durchaus charakteristisch für die Forschungslandschaft zur Proliferation von Klein- und Leichtwaffen. Aufgrund der hohen Dunkelziffer illegaler Verbreitung ist ein insgesamt vorsichtiger Umgang mit Zahlen und Statistiken geboten. Häufig beruhen die Erkenntnisse auf Einzelfallstudien und nach wie vor fehlt es an systematischen und vergleichenden Fallstudien, doch wächst die Erkenntnis, dass Schusswaffengewalt vor allem in Form von Gewaltkriminalität und bewaffneter Gewalt unterhalb der Kriegsschwelle auftritt (Kreutz/Marsh 2012: 43).

Das weltweite Kleinwaffenaufkommen wird gegenwärtig auf 875 Millionen Stück geschätzt. Interessant ist dabei, dass sich nur $25 \%$ dieser Waffen in staatlichem Besitz befinden, also in den Arsenalen von Streitkräften und Polizei. Der größte Teil dieser Waffen ist in den Händen von Privatpersonen, oftmals nicht offiziell registriert und damit auch nicht staatlich kontrolliert. Allein in Deutschland sind 5,7 Millionen Waffen in Privatbesitz registriert - die Zahl der nicht registrierten Waffen wird auf 13 bis 23 Millionen geschätzt (Karp 2014: 71). Neuere Forschungsergebnisse treten auch der Annahme entgegen, dass die illegale Kleinwaf- 
fenproliferation allein ein Problem fragiler Staaten ist. Vielmehr kämpfen durchaus auch westliche Demokratien mit den Folgen illegaler Kleinwaffenverbreitung in Form von Gewaltkriminalität und häuslicher Gewalt (Greene 2014: 267). Allerdings betrachten Forschung und Politik die Kleinwaffenproliferation bislang primär im Kontext von Rüstungskontrolle und Abrüstung. Relativ separiert davon findet sich die Forschung zu privatem Waffenbesitz und Schusswaffengewalt (domestic gun violence) (Batchelor/Muggah 2014: 120; Greene/Marsh 2012: 81-85). 2006 hat der Small Arms Survey mit Unterstützung der Schweiz und Norwegens versucht, beide Bereiche zusammenzuführen. Mit der Geneva Declaration on Armed Violence soll die Kleinwaffenproliferation in den Gesamtkontext der bewaffneten Gewalt eingebettet werden. ${ }^{7}$

Die Gründe für die illegale Kleinwaffenproliferation sind vielfältig - sie beginnt aber nicht selten als legaler, von Staat zu Staat gehandelter Waffenexport (Bourne 2012: 30). Der Small Arms Survey nennt rund 98 Staaten, in denen Kleinwaffen produziert und exportiert werden (SAS 2003: 27). In den vergangenen zehn Jahren ist der globale Handel mit Klein- und Leichtwaffen weltweit um 95\% auf einen Wert von 2,254 Milliarden US-Dollar gestiegen (SAS 2014: 113). ${ }^{8}$ Nach den Vereinigten Staaten von Amerika und Russland wird Deutschland als drittgrößter Exporteur von Klein- und Leichtwaffen genannt (GKKE 2014), im Hinblick auf Kleinwaffenmunition listet der Small Arms Survey Deutschland gar auf Platz 2 nach den Vereinigten Staaten von Amerika (SAS 2014: 116). Zahlreiche Staaten stellen deutsche Kleinwaffen, wie das G3- oder das G36-Sturmgewehr in Lizenz her, darunter etwa Pakistan, Brasilien, die Türkei und Saudi-Arabien. ${ }^{9}$ Problematisch sind Kleinwaffenexporte an Drittstaaten, weil diese oftmals trotz bestehender Endverbleibserklärungen des Empfängerlandes weiter gehandelt werden oder so unzureichend kontrolliert sind, dass sie gestohlen oder von staatlichen Sicherheitskräften verkauft werden. ${ }^{10}$ So warnte erst kürzlich die US-Armee, dass die seit 2004 zum Wiederaufbau an die afghanischen Sicherheitskräfte gelieferten 747.000 AK-47-Sturmgewehre zum Teil unzureichend

7 Siehe dazu www.genevadeclaration.org (letzter Zugriff: 29.7.2014).

8 Der Small Arms Survey mit Sitz in Genf beobachtet seit 2001 den Trend im Kleinwaffenhandel (www.smallarmssurvey.org). Die Zahlen und Statistiken sind auf Grundlage der United Nations Commodity Trade Statistics Database erstellt (SAS 2014: 136). Diese Datenbank erfasst den weltweiten Warenhandel, unter anderem auch alle Waffenkategorien, also auch Pistolen und Handfeuerwaffen. Allerdings weist auch diese Datenbank Lücken auf, denn nicht alle Staaten melden Zahlen zum Waffenhandel bzw. zu allen Kategorien.

9 Ein Überblick hierzu findet sich auch unter http://salw-guide.bicc.de (letzter Zugriff: 29.7.2014).

10 Endverbleibserklärungen sind ein vom Empfängerstaat zu unterschreibendes Dokument, das bescheinigen soll, dass die Waffen in diesem Land verbleiben. In den Vereinigten Staaten von Amerika werden solche Endverbleibserklärungen durch sogenannte post-shipment-Kontrollen zum Teil auch verifiziert. Eine solche Vorgehensweise wird derzeit auch im deutschen Wirtschaftsministerium in Erwägung gezogen. Siehe dazu http://www.bmwi.de/BMWi/Redaktion/PDF/P-R/ruestungsexpor tbericht-2013-geleitwort, property=pdf,bereich=bmwi2012, sprache=de,rwb=true.pdf (letzter Zugriff: 24.9.2014). 
registriert und kontrolliert seien und damit die Gefahr der illegalen Proliferation hoch sei (SIGAR 2014). Schon früher hatte es auch im Zusammenhang mit deutschen Kleinwaffenexporten für den Wiederaufbau der Polizei im Irak und in Afghanistan ähnliche Probleme gegeben. Wie schwierig die Kontrolle staatlicher Waffenbestände gerade auch in Nachkriegssituationen ist und welche Proliferationswege daraus entspringen, zeigt auch das Beispiel Libyen. 2011 fielen den Oppositionskräften fabrikneue G36-Sturmgewehre der libyschen Streitkräfte in die Hände, die vermutlich als legaler Rüstungsexport von Deutschland nach Ägypten genehmigt worden waren. Allerdings waren inzwischen die Seriennummern aus den Kunststoffgehäusen dieser Waffen entfernt worden, was eine Rückverfolgung erschwerte (Grässlin 2013: 195).

Kleinwaffen gelangen jedoch nicht nur über solche Retransfers in unrechtmäßige Hände, auch verdeckte Waffenlieferungen (covert supply) werden häufig zum Problem. Frankreich und die Vereinigten Staaten statteten die libyschen Oppositionskräfte im Geheimen mit Kleinwaffen aus, während die NATO offiziell ein Waffenembargo überwachte (Fehl 2011: 6). Nach Beendigung der Kampfhandlungen und des Machtvakuums in Libyen blieben die Kleinwaffenbestände der ehemaligen Streitkräfte und der Oppositionsverbände zunächst unkontrolliert. Unzählige Waffen aus diesen Beständen sind auch zusammen mit ehemaligen Tuareg-Söldnern nach Mali gelangt und haben dazu beigetragen, dass die bestehende Konfliktsituation dort gewaltsam eskalierte (CAR 2013) ${ }^{11}$ Der Bericht des UN-Generalsekretärs zu Kleinwaffen schlussfolgert, dass die unkontrollierte Verbreitung libyscher Waffen eine ganze Region destabilisiert und grenzübergreifende organisierte Gewaltkriminalität befördert hat - mehr als zwölf Staaten in den Regionen Maghreb, Sahel und Levante sowie am Horn von Afrika sind davon betroffen. Auch nach Gaza und Syrien sind ehemals libysche Waffen gelangt (UNSC 2013: 3). Dies verdeutlicht, wie wichtig eine unmittelbare internationale Kontrolle der Kleinwaffenbestände ehemaliger Streitkräfte und Rebellen nach Beendigung von Kampfhandlungen ist. Gleichzeitig zeigt das Beispiel Libyen, wie folgenreich Kleinwaffenlieferungen an nicht-staatliche Akteure, aber auch reguläre Streitkräfte sein können, wenn diese Waffen unzureichend kontrolliert und gesichert werden - in der Kosten-NutzenAbwägung der Waffenlieferungen an die Kurden im Irakkonflikt hätten diese Erkenntnisse stärker berücksichtigt werden müssen.

11 Die Konfliktursachen in Mali sind vielschichtig und nicht Thema dieses Artikels. Vielmehr soll das Beispiel nur die Problematik der Proliferation von unkontrollierten Kleinwaffen in Nachkriegssituationen und die Gefahr der Regionalisierung von Konflikten verdeutlichen, die gerade die Region Subsahara-Afrika immer wieder betrifft. Siehe dazu Buro/Ronnefeldt (2013) sowie die Ausgabe 2/2014 der Zeitschrift Sicherheit + Frieden mit dem Schwerpunkt »Intervention zwischen Krieg und Ethik: Das Beispiel Mali«. 
Ein Großteil der weltweit illegal gehandelten Klein- und Leichtwaffen sind älteren Baujahrs. Aufgrund ihrer hohen Lebensdauer stammen solche Waffen noch aus Zeiten des Ost-West-Konflikts und der großzügigen Ausstattung von Klientelstaaten mit Waffen durch die beiden Supermächte. Nach 1989 reduzierten die Bündnisstaaten des ehemaligen Warschauer Paktes und der NATO ihre Streitkräfte sowie deren Bewaffnung: Solche überschüssigen Waffen und auch Munition gelangten auf legalem oder illegalem Weg in potenzielle Konfliktgebiete. Die Praxis der Militärhilfe und verdeckter Waffenlieferungen hält auch nach Ende des Ost-WestKonflikts weiter an. Im Kampf gegen den Terror setzen die Vereinigten Staaten von Amerika auf Militärausbildungsprogramme und flankieren diese mit Waffenlieferungen an neue strategisch relevante Bündnispartner. Russland unterstützte Rebellen in Moldawien und Georgien mit Kleinwaffen. Verdeckte staatliche Lieferungen an nicht-staatliche Akteure (covert supply) werden auch von Staaten des Mittleren Ostens betrieben. Iran, Libyen, Irak und Syrien haben immer wieder Klein- und Leichtwaffen an die Gruppen Hamas, Abu Nidal und Hisbollah geliefert (SAS 2003: 130). Munition aus chinesischer, iranischer und sudanesischer Produktion nach 2000 ist wiederholt in Konfliktgebieten gefunden worden, etwa in der Elfenbeinküste, Syrien oder im Südsudan (SAS 2014: 194-95; Anders 2014; CAR 2013). Staaten umgehen vorhandene UN-Embargos und liefern Waffen und Munition an Konfliktparteien (Anders 2014). Ein Beispiel jüngerer Vergangenheit sind AK-47Waffenlieferungen aus Eritrea und dem Iran an die Verbände der Islamic Court Union in Somalia. UN-Berichte dokumentieren die Vielzahl der Verstöße: Äthiopien lieferte Munition an die Übergangsregierung; Ägypten unterstützte die nichtstaatlichen Gruppierungen mittels militärischem Training (UNSC 2006: 9-31). Doch es sind nicht nur Staaten, die UN-Embargos brechen. Ein Teil des Geschäfts in Konfliktgebieten geht zulasten professioneller Waffenhändler und ihrer Netzwerke - oftmals steht der illegale Waffenhandel in engem Zusammenhang mit anderen kriminellen Praktiken wie Drogen- oder Diamantenhandel. Professionelle Waffenhändler halfen etwa den Konfliktparteien im ehemaligen Jugoslawien, trotz bestehender UN-Waffenembargos ihren Nachschub an Kleinwaffen zu beschaffen und Luftfrachtunternehmen liefern illegal Waffen nach Somalia (Feinstein 2012: 123).

\section{Die hohen Kosten der illegalen Kleinwaffenproliferation}

Bewaffnete Gewalt verübt mit Kleinwaffen hat viele Gesichter - von schweren Menschenrechtsverletzungen durch Polizeigewalt über organisierte Gewaltkriminalität in Zusammenhang mit Drogen- oder Menschenhandel zu Gewalt durch 
Straßengangs, Terrorismus oder häusliche (Waffen-)Gewalt gegen Frauen und Mädchen. Die Forschung differenziert zumeist zwischen den direkten und indirekten Folgekosten von Kleinwaffengewalt. Von direkten Folgekosten ist die Rede im Fall tödlicher und nichttödlicher Verletzungen - aber auch die Kosten der Gesundheitsversorgung und Opportunitätskosten, wie Verdienstausfälle, werden einkalkuliert (Batchelor/Muggah 2014: 118). Staaten mit der höchsten Gewaltrate durch Schusswaffen sind neben El Salvador Jamaika und der Irak. Gewaltkriminalität und organisierte Gewalt sind vor allem in Zentral- und Südamerika deutlich stärker ausgeprägt als in Europa oder Asien. Demographisch gesehen, sind vor allem junge Männer zwischen 15 und 29 Jahren die primären Opfer von Schusswaffengewalt (SAS 2008: 219). Geschlechtsspezifische Datenauswertungen ergeben einen starken Zusammenhang zwischen Waffenbesitz und dem tödlichen Ausgang häuslicher Gewalt (SAS 2004: 183). ${ }^{12}$ Allein 66.000 Frauen werden weltweit jedes Jahr Opfer von Schusswaffengewalt in Staaten, die nicht unmittelbar in kriegerische Konflikte verwickelt sind (Geneva Declaration Secretariat 2011). Vor allem der Forschungszweig zu privatem Waffenbesitz und dessen Regulierung in den Vereinigten Staaten von Amerika hat einen »robusten Befund « des unmittelbaren Zusammenhangs zwischen Schusswaffenbesitz und hohen Selbstmordraten belegt. Dagegen sehen die Forscher das Verhältnis von Waffenverfügbarkeit und Gewaltrate zunächst nur als Korrelation, da dieser Zusammenhang wesentlich komplexer sei (Greene/Marsh 2012: 84).

Ungleich schwerer zu bewerten sind die indirekten Folgekosten von Schusswaffengewalt. Zunächst beeinträchtigt ein hohes Kleinwaffenaufkommen die gefühlte individuelle Sicherheit der Menschen, aber auch der Dörfer oder Städte. Betroffen sind Gesundheitssysteme und Bildungseinrichtungen wie Schulen, die von Kindern nicht mehr besucht werden können, wenn sich die Sicherheitssituation verschlechtert (SAS 2003: 140). ${ }^{13}$ Studien zeigen, dass Städte und Großstädte stärker von Gewaltkriminalität betroffen sind und sich dies vor allem in Ländern mit einem niedrigen Human Development Index zeigt. Oftmals sind UN-Friedensmissionen oder humanitäre Hilfseinsätze aufgrund von Waffengewalt in Konflikten oder auch in Nachkriegssituationen nicht in der Lage, mit Hilfslieferungen zu Flüchtlingen vorzudringen (SAS 2002: 186-189). Die indirekten Folgekosten der illegalen Kleinwaffenverbreitung sind vielfältig: Unter anderem wird die nachhaltige und lang-

12 Eine wissenschaftliche Studie in 25 Staaten mit hohem Einkommen zeigt, dass die Wahrscheinlichkeit, dass Frauen Opfer tödlicher Schusswaffengewalt werden, in den Vereinigten Staaten von Amerika zwölfmal höher ist als in den übrigen 24 Ländern (SAS 2014: 13).

13 Eine Weltbankstudie zu Jamaika zeigt, dass 30\% der Mädchen nicht zur Schule gehen, weil sie sich vor der alltäglichen Gewaltkriminalität in ihren Distrikten fürchten (SAS 2003: 141). 
fristige Entwicklungszusammenarbeit in ohnehin fragilen Staaten beeinträchtigt. Die Weltbank verweist darauf, dass Länder mit hoher Waffengewalt negative Wachstumsraten aufweisen und ausländische Direktinvestitionen ausbleiben - die Opportunitätskosten sind insgesamt hoch (World Bank 2001).

\section{Globale Regelungen der Kleinwaffenkontrolle und ihre Aussichten auf Erfolg}

Die Regelungen zur Bekämpfung der unrechtmäßigen Verbreitung von Klein- und Leichtwaffen sind auf globaler Ebene noch relativ überschaubar, dagegen sind gerade auf regionaler und subregionaler Ebene eine Vielzahl von Verträgen, Abkommen und Aktionsprogrammen in den vergangenen 15 Jahren verabschiedet worden (Garcia 2014: 236-254). Die Kleinwaffenkontrolle ist Teil der humanitären Rüstungskontrolle (Wisotzki 2013 a: 91-96). ${ }^{14}$ Nachdem 1997 mit dem Anti-Personenminenvertrag eine wichtige Weiche gestellt worden war, konzentrierten sich die Staaten im Rahmen der Vereinten Nationen in den kommenden Jahren auf die Kleinwaffenproblematik - 2001 konnte das UN-Kleinwaffenaktionsprogramm verabschiedet werden. Darin sind alle Staaten aufgefordert, die staatlichen Waffenbestände der Streitkräfte und der Polizei zu registrieren und zu kontrollieren, um ihre Proliferation zu verhindern und überschüssige Waffen zu vernichten. Herstellung, Handel, Transfer und Besitz von Kleinwaffen sollen, sofern staatlich nicht genehmigt, geahndet und unter Strafe gestellt werden. Von starker Kleinwaffenproliferation betroffene Staaten haben die Möglichkeit, Unterstützung durch westliche Geberländer zu erhalten.

Mehr als zehn Jahre nach seiner Beschlussfassung hat das PoA zur Sensibilisierung und Bewusstseinsbildung für die Problematik der unrechtmäßigen Verbreitung von Kleinwaffen beigetragen. Auch sind neue Normen und Regelungen entstanden. Beispielhaft sei etwa das internationale Instrument zur Markierung und Nachverfolgung (International Tracing Instrument, ITI) von 2005 genannt, das darauf abzielt, die internationale Kooperation zwischen den Strafverfolgungsbehörden zu verbessern und länderspezifische Markierungsstandards einzuführen (SAS 2011: 49). ${ }^{15}$ Die Abrüstungsabteilung der Vereinten Nationen hat ein Unterstützungssystem zur Implementierung des PoA eingerichtet, das bereits in vielen Staaten zu

14 Charakteristisch für die humanitäre Rüstungskontrolle ist der Fokus auf menschliche Sicherheit, oftmals auch auf Opferrehabilitation und Unterstützung betroffener Staaten durch die Geberländer.

15 Das Feuerwaffenprotokoll (firearms protocol) von 2001 ergänzt das PoA, indem es sich auf Aspekte der Verbrechensbekämpfung und der Kleinwaffenproliferation konzentriert. Siehe dazu http://www.unodc.org/unodc/en/firearms-protocol/firearmsprotocol.html (letzter Zugriff: 24.9.2014). 
Fortschritten in Gesetzgebung und administrativen Verfahren geführt hat. ${ }^{16}$ Die regelmäßigen Staatentreffen und die bislang zwei Überprüfungskonferenzen 2006 und 2012 haben jedoch gezeigt, dass es starke Diskrepanzen zwischen den freiwilligen, wenig selbstkritischen Staatenberichten und der Realität in von Kleinwaffenproliferation betroffenen Staaten gibt. Allein die Sicherung staatlicher Waffenbestände bleibt in vielen Regionen begrenzter Staatlichkeit weiterhin ein Problem. Viele Ziele des PoA sind nicht erreicht, wie etwa zentralisierte Datenerfassung von staatlichen Kleinwaffenbeständen in zahlreichen Staaten oder deren Registrierung und Markierung (SAS 2011: 59). Deutschland ist international federführend in den Bemühungen, das PoA normativ zu stärken und umzusetzen. Dabei ist Deutschland einer der führenden Geberstaaten und unterstützt sowohl finanziell als auch durch technische Expertise, z. B. durch Seminare zur Markierung und Sicherheit von Kleinwaffen durch das Zentrum für Verifikationsaufgaben der Bundeswehr in Geilenkirchen.

Kritiker bemängelten lange Zeit, dass das PoA zu stark auf die unkontrollierte, illegale Proliferation fokussiert sei, den legalen staatlichen Handel mit Kleinwaffen insgesamt aber unberücksichtigt lasse. Diese Lücke sucht nun seit April 2013 der Vertrag zur Kontrolle des Waffenhandels (ATT) zu schließen. ${ }^{17}$ Der Vertrag verfolgt zwei Ziele: Der globale konventionelle Waffenhandel soll erstens reguliert werden, um zweitens den illegalen, unkontrollierten Handel einzudämmen. Hierzu hat die internationale Staatengemeinschaft gemeinsame Kriterien verhandelt. Verboten sind Transfers ${ }^{18}$ nach Artikel 6, wenn sie gegen UN-Waffenembargos verstoßen oder wenn die Waffen zur Begehung von Völkermord, zu Verbrechen gegen die Menschlichkeit, zu schweren Verletzungen der Genfer Abkommen von 1949 oder sonstigen Kriegsverbrechen beitragen. Letztlich wird damit jedoch nur ohnehin schon bestehendes Völkerrecht festgeschrieben. Artikel 7 identifiziert dann jedoch weitere Bewertungskriterien für Rüstungstransfers, etwa Frieden und Sicherheit, humanitäres Völkerrecht, Menschenrechte, geschlechtsspezifische Gewalt, Terrorismusgefahren und Risiken des organisierten Verbrechens. Artikel 11 benennt ein Paket an Maßnahmen, um Umleitungsgefahren bei Waffentransfers zu minimieren, beispielsweise in Form einer Risikobewertung im Vorfeld der Genehmigung. In-

16 Siehe dazu http://www.poa-iss.org (letzter Zugriff: 24.9.2014). Hier finden sich auch die Texte des PoA und des ATT.

17 Der Geltungsbereich des Vertrages umfasst neben den sieben Kategorien des UN-Waffenregisters (Kampfpanzer, gepanzerte Kampffahrzeuge, großkalibrige Artilleriesysteme, Kampfflugzeuge, Kampfhubschrauber, Kriegsschiffe, Raketen einschließlich ihrer Start- und Abschusssysteme) auch Klein- und Leichtwaffen.

18 Unter die Bezeichnung »Transfer« fallen Ausfuhr, Einfuhr, Durchfuhr sowie Umladung und Vermittlungstätigkeiten. 
zwischen haben 121 Staaten den ATT unterzeichnet und 53 ihn ratifiziert, darunter auch die großen Rüstungsexporteure. Deutschland hat ihn als einer der ersten Staaten weltweit ratifiziert, die Vereinigten Staaten von Amerika haben ihn unterschrieben, jedoch ist fraglich, ob der US-Senat ihn auch ratifizieren wird. Da nun über 50 Staaten eine Ratifikationsurkunde bei den UN hinterlegt haben, kann der ATT in 90 Tagen in Kraft treten (GKKE 2013; Wisotzki 2013 b).

$\mathrm{Ob}$ und inwieweit der ATT einen Beitrag zur Lösung der unkontrollierten Kleinwaffenproliferation leisten kann, wird zum einen von der Universalität seiner Mitgliedschaft abhängen, aber auch von der Implementierungsbereitschaft der Mitgliedsstaaten. Schon während der zwei Verhandlungsrunden artikulierten Staaten ihren Widerstand: Iran, Syrien und Nordkorea ließen die Verhandlungen im März 2013 scheitern. Zwar erzielte der ATT bei der Abstimmung vor der UN-Generalversammlung am 2. April 2013 die nötige Zweidrittelmehrheit, doch enthielten sich wichtige Rüstungsexporteure und Schwellenländer wie Russland, China, Indien, Indonesien, Pakistan und die arabischen Staaten. In der Definition konventioneller Rüstungsgüter konnten sich die Staaten nur auf einen Minimalstandard einigen. Aufgrund des Widerstands der Vereinigten Staaten sind Munition sowie Rüstungskomponenten und -bauteile schwächer verregelt als die übrigen Rüstungsgüter. Auch fehlt mit dem Hinweis auf »neue Technologien« eine Norm, die den ATT und seine Mitgliedsstaaten dazu befähigt, dynamisch auf technologische Fortschritte im globalisierten Rüstungssektor zu reagieren. Noch steckt die Technologie in den Kinderschuhen, doch können schon jetzt einfache Schusswaffen und ihre Komponenten mit Hilfe von 3D-Druckern gefertigt werden (Mack 2014: 102-107; UNGA 2014: 4-5). Herzstück des ATT sind die Kriterien, nach denen der Handel mit konventionellen Rüstungsgütern künftig reguliert werden soll. Doch mangelt es den Kriterien nicht nur an Präzision, sondern der Grad der Verpflichtung geht insbesondere für westliche Staaten nicht über das hinaus, was ohnehin durch nationale und regionale Gesetzgebung abgedeckt ist. ${ }^{19}$ Auch sieht der ATT keinerlei Sanktionen bei Nichtbeachtung vor, einziges Verifikationsinstrument ist die jährliche, nicht-öffentliche Berichtspflicht der Mitgliedsstaaten (SAS 2014: 77-107).

\section{Fazit}

Die weltweite Verbreitung von Kleinwaffen stellt die internationale Staatengemeinschaft weiterhin vor beträchtliche Herausforderungen - die Anzahl illegaler Kleinwaffen weltweit ist unermesslich hoch und das Proliferationsrisiko dieser

19 So sind die Kriterien des Gemeinsamen Standpunktes der EU für die Kontrolle der Ausfuhr von Militärtechnologie und Militärgütern aus dem Jahr 2008 weitreichender als die des ATT. 
Waffen ist beträchtlich. Umso bedeutsamer ist es, dass Deutschland als einer der größten Rüstungsexporteure eine verantwortungsvolle Politik betreibt. Deutschland hat sich mit den eigenen Politischen Grundsätzen für den Export von Kriegswaffen und sonstigen Rüstungsgütern aus dem Jahr 2000 einer restriktiven Rüstungsexportpolitik verschrieben. Ähnlich wie der rechtlich verbindliche Gemeinsame Standpunkt der EU zu gemeinsamen Regeln für die Kontrolle der Ausfuhr von Militärtechnologie und -gütern von 2008 benennen auch die Politischen Grundsätze Kriterien für eine Bewertung von Rüstungsausfuhren. Genehmigungen für Kriegswaffen werden nicht erteilt, wenn die Empfängerländer in bewaffnete Auseinandersetzungen verwickelt sind oder solche drohen. Keine Waffen sollten auch an solche Länder geliefert werden, die gegen Endverbleibserklärungen verstoßen oder Terrorismus unterstützen. Bewertet werden aber auch, inwieweit die Empfängerländer solcher Rüstungsexporte Maßnahmen der Nichtverbreitung, Rüstungskontrolle und Abrüstung unterstützen. ${ }^{20}$

Die oben genannten Beispiele der Drittstaaten, an die Deutschland in jüngster Vergangenheit Kleinwaffen und Munition geliefert hat, fordern ein kritisches Nachfragen heraus. In den Politischen Grundsätzen wird betont, dass keine Rüstungsgüter geliefert werden dürfen, wenn bewaffnete Auseinandersetzungen, die Gefahr eines Missbrauchs zur inneren Repression oder fortdauernde Menschenrechtsverletzungen im Empfängerland zu verzeichnen sind. Wendet man diesen Kriterienkatalog so restriktiv an, wie es in den Politischen Grundsätzen postuliert wird, hätte es 2008 keine Lizenz für die G36-Herstellung in Saudi-Arabien geben dürfen.

Statt jedoch stets nur einzelne Beispiele einer verfehlten deutschen Rüstungsexportpolitik zu diskutieren, erscheint es vielmehr erforderlich, ein generelles Umdenken in der deutschen Rüstungsexportpolitik einzuleiten - auch um bestehende und neue Regelungen, wie den ATT, nicht schon vor seinem Inkrafttreten sogleich zum Papiertiger zu degradieren. Gerade wegen ihrer potenziellen Auswirkung auf Menschenrechte und aufgrund ihres hohen Proliferationsrisikos während und nach Konflikten muss es für den Export von Kleinwaffen und Munition sowie für die Lizenzvergaben eine grundsätzliche Begründungspflicht für den Transfer an Drittstaaten geben, die außen-, sicherheits- und friedenspolitische Gründe für die Ausfuhr detailliert benennt. In der Abwägung sind die möglichen Kosten von Menschenrechtsverletzungen im Empfängerland schwerer zu gewichten als sicherheitsund stabilitätspolitische Kalküle. Wer als Empfängerland gegen die Endverbleibserklärungen verstößt und deutsche Kleinwaffen re-exportiert, sollte mit Konse-

20 Siehe dazu https://www.bmwi.de/BMWi/Redaktion/PDF/A/aussenwirtschaftsrecht-grundsaetze, property=pdf,bereich=bmwi2012, sprache $=\mathrm{de}, \mathrm{rwb}=$ true.pdf (letzter Zugriff: 24.9.2014). 
quenzen zu rechnen haben - das sollte im Übrigen auch für NATO- und EU-Staaten gelten. Um dem Prinzip der Endverbleibserklärungen mehr Gewicht zu verleihen, müssen letztlich zumindest selektiv post-shipment-Kontrollen stattfinden. Das Beispiel der aus den Kunststoffgehäusen entfernten Seriennummern der in Libyen aufgetauchten G36-Gewehre hat zudem gezeigt, wie unzureichend selbst die deutschen Markierungsstandards von Kleinwaffen sind. Sturmgewehre für die Bundeswehr werden auch auf dem Stahl des Waffenlaufs markiert, ein Versuch der Entfernung würde die Waffe unbrauchbar machen. Ein solcher Standard sollte für Kleinwaffenproduktion »made in Germany « generell eingeführt werden. ${ }^{21}$ Er sollte auch in die in Aussicht gestellten »Kleinwaffengrundsätze« aufgenommen werden, die von der Bundesregierung derzeit erarbeitet werden (BMWi 2014 b: 7). Sie sollten im Übrigen Gesetzescharakter erhalten, damit künftige Regierungen nicht erneut hinter einen mühevoll erarbeiteten Standard zurückfallen.

Mehr Transparenz statt einer Politik im Verborgenen des Bundessicherheitsrates tut Not. Halbjährliche Rüstungsexportberichte und raschere Informationen an den Wirtschaftsausschuss des Deutschen Bundestages sind ein Anfang, aber nicht ausreichend als parlamentarisches Kontrollsystem in einer Demokratie. Deutschland braucht eine wirkungsvolle Debatte über die Zukunft deutscher Rüstungsexporte, an der die relevanten Akteure - Ministerien, Bundestagsabgeordnete, Rüstungsindustrie, Gewerkschaften, Wissenschaft und Zivilgesellschaft - beteiligt werden. Eine solche Debatte über deutsche Rüstungsexporte ergänzt gleichzeitig den Review 2014 über die Zukunft der deutschen Außenpolitik.

Deutschland engagiert sich international federführend in der Umsetzung des UNKleinwaffenaktionsprogramms. Das Aktionsprogramm zielt vor allem auf die Bekämpfung des unrechtmäßigen Kleinwaffenhandels ab. Mit dem ATT wird nun eine wichtige Regelungslücke geschlossen, da nun auch die legalen Staat-zu-StaatLieferungen konventioneller Rüstungsgüter global verbindlichen Standards unterworfen werden. Deutschland hat den ATT als einer der ersten Staaten schon im Juni 2013 ratifiziert, auch um damit den Stellenwert des Vertrages zu unterstreichen. In der Konsequenz sollte eine restriktive deutsche Rüstungsexportpolitik auch darauf abzielen, keine Klein- und Leichtwaffen mehr an solche Drittstaaten zu liefern, die sich dem ATT und den Meldungen an das UN-Waffenregister verweigern. Die neuen »Kleinwaffengrundsätze« könnten hier ein wichtiges Signal setzen, indem sie eine solche Norm aufnehmen. Auf diese Weise könnte die Bundesregierung dem

21 Die SPD-Fraktion des Deutschen Bundestags hatte 2012 hierzu schon einmal einen Antrag zur verbesserten Markierung von Klein- und Leichtwaffen sowie Munition eingebracht. Siehe dazu die Drucksache 17/11876 des Deutschen Bundestages, einsehbar unter: http://dipbt.bundestag.de/ doc/btd/17/118/1711876.pdf (letzter Zugriff: 12.12.2012). 
Ziel der Universalität solcher Regelungen Nachdruck verleihen und wirkungsvoll unterstreichen, dass sie eine verantwortungsvolle Politik konventioneller Rüstungskontrolle verfolgt, in der auch der Waffenhandel zum festen Bestandteil wird. Eine Politik, die internationales Recht an entscheidenden Stellen einfordert und gestaltet, sollte nicht durch eine auf nationaler Ebene widersprüchliche Exportpolitik konterkariert werden, die auf internationaler Ebene die eigene politische Glaubwürdigkeit verspielt.

\section{Literatur}

Anders, Holger 2014: Identifying Sources. Small-calibre Ammunition in Cote D'Ivoire (Special Report, Small Arms Survey, June 2014), Genf.

Batchelor, Peter/Muggah, Robert 2014: Effects: An Emerging Research and Policy Agenda, in: Batchelor, Peter/Kenkel, Kai Michael (Hrsg.): Controlling Small Arms. Consolidation, Innovation and Relevance in Research and Policy, London, 118-149.

BMWi - Bundesministerium für Wirtschaft und Energie 2014 a: Bericht der Bundesregierung über ihre Exportpolitik für konventionelle Rüstungsgüter im Jahre 2013. Rüstungsexportbericht 2013, in: http://www.bmwi.de/BMWi/Redaktion/ $\mathrm{PDF} /$ Publikationen/ruestungsexportbericht-2013, property=pdf,bereich=bmwi2 012, sprache=de,rwb=true.pdf; 4.9.2014.

BMWi - Bundesministerium für Wirtschaft und Energie 2014 b: Bericht der Bundesregierung über ihre Exportpolitik für konventionelle Rüstungsgüter im ersten Halbjahr 2014, in: http://www.bmwi.de/BMWi/Redaktion/PDF/Publikationen/ ruestungsexportbericht-zwischenbericht-2014, property=pdf,bereich=bmwi201 2,sprache $=$ de,rwb=true.pdf; 24.10.2014.

Bourne, Mike 2012: Small Arms and Light Weapons Spread and Conflict, in: Greene, Owen/Marsh, Nicholas (Hrsg.): Small Arms, Crime and Conflict. Global Governance and the Threat of Armed Violence, London, 29-42.

Brost, Marc/Friedrichs, Hauke/Pinzler, Petra 2014: Frieden durch deutsche Waffen?, in: Die Zeit 34, 14.8.2014, 6.

Buro, Andreas/Ronnefeld, Clemens 2013: Dossier VI. Der Mali-Konflikt oder: Der

Kampf um die Kontrolle von Nord- und Westafrika, in: http://www.friedenskooperative.de/gifs/dossier6.pdf; 30.9.2014.

CAR-ConflictArmamentResearch2013:RebelForcesinNorthernMali.Documented

Weapons, Ammunitions and Related Material, in: http://www.smallarmssurvey.org/fileadmin/docs/E-Co-Publications/SAS-SANA-Conflict-ArmamentResearch-Rebel-Forces-in-Northern-Mali.pdf; 15.1.2014. 
Fehl, Caroline 2011: Ein hausgemachtes Dilemma. Der Bürgerkrieg in Libyen erteilt Europa unangenehme Lehren (HSFK-Standpunkt 7/2011), Frankfurt a. M.

Feinstein, Andrew 2012: Waffenhandel. Das globale Geschäft mit dem Tod, Hamburg.

Garcia, Denise 2014: The Evolution and Consolidation of Norms on Small Arms, in: Batchelor, Peter/Kenkel, Kai Michael (Hrsg.): Controlling Small Arms. Consolidation, Innovation and Relevance in Research and Policy, London, 236-254.

Geneva Declaration Secretariat 2011: Global Burden of Armed Violence 2011. Lethal Encounters, Cambridge.

GKKE - Gemeinsame Konferenz Kirche und Entwicklung 2014: Rüstungsexportbericht 2013 der GKKE, Bonn.

Grässlin, Jürgen 2013: Schwarzbuch Waffenhandel. Wie Deutschland am Krieg verdient, München.

Grebe, Jan 2013: Deutsche Rüstungsexporte: Zwischen mangelnder Transparenz, der Notwendigkeit parlamentarischer Kontrolle und einer Neujustierung der Rüstungsexportpolitik, in: Zeitschrift für Friedens- und Konfliktforschung 2: 2, 279-289.

Greene, Owen 2014: Small Arms Research: Dynamics and Emerging Challenges, in: Batchelor, Peter/Kenkel, Kai Michael (Hrsg.): Controlling Small Arms. Consolidation, Innovation and Relevance in Research and Policy, London, 257-285.

Greene, Owen/Marsh, Nicholas 2012: Armed Violence within Societies, in: dies. (Hrsg.): Small Arms, Crime and Conflict. Global Governance and the Threat of Armed Violence, London, 79-104.

Karp, Aron 2014: Stockpiles: The Global Geography of Small Arms Numbers, in: Batchelor, Peter/Kenkel, Kai Michael (Hrsg.): Controlling Small Arms. Consolidation, Innovation and Relevance in Research and Policy, London, 64-82.

Kreutz, Joakim/Marsh, Nicholas 2012: Lethal Instruments: Small Arms and Deaths in Armed Conflict, in: Greene, Owen/Marsh, Nicholas (Hrsg.): Small Arms, Crime and Conflict. Global Governance and the Threat of Armed Violence, London, 43-63.

Mack, Daniel 2014: What Next? Thoughts for Global Civil Society Working on Arms Control and Armed Violence Reduction, São Paulo.

Marsh, Nicholas 2012: The Tools of Insurgency. A Review of the Role of Small Arms and Light Weapons in Warfare, in: Greene, Owen/Marsh, Nicholas (Hrsg.): Small Arms, Crime and Conflict. Global Governance and the Threat of Armed Violence, London, 13-28. 
SIGAR - Special Inspector General for Afghanistan Reconstruction 2014: Afghan National Security Forces: Actions Needed to Improve Weapons Accountability (SIGAR 14-84 Audit Report), Virginia.

SAS - Small Arms Survey 2003: Small Arms Survey 2003. Development Denied, Oxford.

SAS - Small Arms Survey 2004: Small Arms Survey 2004. Rights at Risk, Oxford. SAS - Small Arms Survey 2008: Small Arms Survey 2008. Risk and Resilience, Oxford.

SAS - Small Arms Survey 2011: Small Arms Survey 2013. States of Security, Oxford.

SAS - Small Arms Survey 2013: Small Arms Survey 2013. Everyday Dangers, Oxford.

SAS - Small Arms Survey 2014: Small Arms Survey 2014. Women and Guns, Oxford.

UNGA - United Nations General Assembly 2014: Report of the Secretary General. Recent Developments in Small Arms and Light Weapons Manufacturing, Technology and Design (A/CONF.192/BMS/2014/1, 6.5.2014), in: http://daccessdds-ny.un.org/doc/UNDOC/GEN/N14/322/93/PDF/N1432293.pdf?OpenElem ent; 9.9.2014.

UNSC - United Nations Security Council 2006: Letter Dated 21 November 2006 from the Chairman of the Security Council Committee established pursuant to Resolution 751 (1992) concerning Somalia addressed to the President of the Security Council (S/2006/913, 22.11.2006), in: http://fas.org/asmp/resources/ govern/109th/S2006913.pdf; 9.9.2014.

UNSC - United Nations Security Council 2013: Report of the Secretary General on Small Arms (S/2013/503, 22.8.2013), in: http://www.securitycouncilreport.org/ atf/cf/\%7B65BFCF9B-6D27-4E9C-8CD3-CF6E4FF96FF9\%7D/s_2013 503.pdf; 9.9.2014.

Wisotzki, Simone 2013 a: Humanitarian Arms Control: The Anti-Personnel Mine Ban Treaty, the Programme of Action on Small Arms and Light Weapons, and the Convention on Cluster Munition, in: Müller, Harald/Wunderlich, Carmen (Hrsg.): Norm Dynamics in Multilateral Arms Control. Interests, Conflicts and Justice, London, 82-106.

Wisotzki, Simone 2013 b: Rüstungsexporte unter verschärfter Kontrolle? Eine Bewertung des internationalen Waffenhandelsvertrags (HSFK-Report 6/2013), Frankfurt a. M. 
World Bank 2001: Development Cooperation and Conflict (World Bank Operational Policies OP 2.30), in: http://web.worldbank.org/WBSITE/EXTERNAL/ PROJECTS/EXTPOLICIES/EXTOPMANUAL/0,,contentMDK:20129203 isCURL:Y menuPK:64701637 pagePK:64709096 piPK:64709108 theSite PK:502184 isCURL:Y,00.html; 9.9.2014.

\section{Die Autorin}

Dr. Simone Wisotzki ist wissenschaftliche Mitarbeiterin des Leibniz-Instituts Hessische Stiftung Friedens- und Konfliktforschung (HSFK) in Frankfurt a. M.

E-Mail: wisotzki@hsfk.de 